\title{
PÚRPURA TROMBOCITOPÉNICO IDIOPÁTICO Y EMBARAZO. CESÁREA VERSUS PARTO VAGINAL
}

\author{
Sergio Hott A. ${ }^{a}$, Alessandro Queirolo S. ${ }^{a}$, Nicolás Veas P.a, Gloria Rubio A. ${ }^{1}$ \\ ${ }^{1}$ Servicio de Hematología, Hospital Militar.
}

anternos de Medicina, Universidad de los Andes.

\section{RESUMEN}

Si bien el púrpura trombocitopénico idiomático (PTI) es una patología poco frecuente, su importancia radica en que existe una alta morbimortalidad materno-fetal durante el embarazo, el parto y el puerperio. Es en el parto donde actualmente existe mayor controversia, con respecto a que vía es más recomendable, cesárea o vía vaginal. Nuestro propósito es acortar la brecha de la duda con respecto a ésta incógnita en base a la literatura disponible actualmente tanto a nivel nacional como internacional presentando una revisión bibliográfica sobre el PTI en el embarazo dando una pequeña reseña sobre la fisiopatología de ésta, diagnóstico y tratamiento, centrándonos en la controversia sobre que vía de parto es la más indicada. Se adoptó la estrategia de búsqueda desarrollada en la revisión electrónica de bases de datos, bajo criterios definidos que permitieron identificar los estudios con mejor evidencia posible. En nuestra búsqueda se encontraron 18 trabajos que cumplieran con nuestros criterios de búsqueda. En la literatura no encontramos un trabajo prospectivo randomizado con un buen grado de evidencia y fuerza que afirme una vía por sobre otra. Los trabajos encontrados corresponden a reportes de casos y guías de expertos que recomiendan un manejo por sobre otro. Podemos concluir que la vía del parto se definiría según condiciones obstétricas y no por el nivel de plaquetas fetales encontrados. Además, algunos de los procedimientos para determinar los niveles de plaquetas estarían relacionado a un mayor riesgo de morbimortalidad que la patología base.

\section{PALABRAS CLAVES: Púrpura trombocitopénico idiopático, plaquetas, cesárea, parto vaginal}

\section{SUMMARY}

The idiopathic throbocytopenic purpura (ITP)is a rare pathology but it's importance lies in the fact that exists a high morbimortality during pregnancy, labor and puerperium. There's a controversy about which methods for labor are more recommended nowadays, either vaginal way or through cesarean and for that purpose this investigation will try to shorten that breach of doubt using both national and international literature giving a closer look to the ITP and it's physiopathology, diagnose and treatment. The strategy used consisted on an electronic review of data bases under defined criterion that allowed us to identify the researches with good evidence. We found eighteen investigations under this criterion. On literature we didn't found any researches with good grade of evidence that probe that one way is more beneficial than the other. Our researches found are cases and guidelines of experts that do recommend one way over the other. The conclusions of our investigation are that the ways of labor will be defined depending on obstetric conditions and not by the level of fetal platelets found. Some of the procedures to determine these levels would be related to a higher morbimortality risk than the IPT per se.

KEY WORDS: Idiopathic thrombocytopenic purpura, platelets, cesarean, vaginal delivery 


\section{INTRODUCCIÓN}

El Púrpura Trombocitopénico Idiopático (PTI) es una afección hematológica infrecuente, que presenta una incidencia de 3,2 casos en 1.000.000. Se define como trombocitopenia aislada con médula ósea normal en ausencia de otras causas de trombocitopenia, como enfermedades autoinmunes, reacciones adversas a drogas, síndrome antifosfolípido o enfermedades virales.

Existe una disminución de las plaquetas circulantes por destrucción de estas, mediada por anticuerpos tipo IgG contra las glicoproteínas de membrana. Este complejo antígeno/anticuerpo es captado por el sistema retículo endotelial, especialmente en el bazo con su consecuente remoción. El anticuerpo materno puede atravesar la barrera placentaria y llegar a la circulación fetal. Es importante tener en cuenta que el nivel de anticuerpos en el suero materno no predice si el feto esta afectado, debido a que la composición antigénica de la superficie fetal es distinta a la de la madre, pudiendo pasar al feto sin afectarlo $(1,2,3)$.

En adultos, la forma de presentación más frecuente es la forma crónica, hasta un $90 \%$ de los casos no presenta recuperación y suele existir recidiva. Esta forma se presenta con mayor frecuencia en mujeres (mujer:hombre=2,6:1) en edad fértil. Es por esta razón que un gran porcentaje de esta patología se diagnostica durante el embarazo, tanto por exámenes de rutina como por exacerbación a causa del embarazo. La aparición de síntomas va a depender del nivel plaquetario que posea la paciente $(2,4)$.
Durante años se ha desconocido cual es la vía del parto más segura para estas pacientes. En el presente trabajo se presenta una revisión bibliográfica sobre el PTI en el embarazo dando una pequeña reseña sobre la fisiopatología de ésta, diagnóstico y tratamiento, centrándonos en la controversia sobre que vía de parto es la más indicada.

Para este trabajo se adoptó la estrategia de búsqueda de información con respecto al PTI y su asociación con la vía del parto, desarrollando una revisión electrónica de bases de datos, que permitió identificar los estudios con mejor evidencia posible. Las bases de datos son las siguientes: medline, embase, healthstar, dare, cochrane, biosis, online medical, database, ebsco, blood, medline plus, pubmed, proquest, además se utilizó cedip.cl, puc.cl.

Los niveles de evidencia y fuerza de las recomendaciones se clasificaron de acuerdo a la North of England Evidence Based Guideline Development Project (Tabla I).

\section{Fisiopatología del PTI y su relación con el em- barazo}

En el PTI el mecanismo de la trombocitopenia es la reducción de la vida de las plaquetas por una destrucción aumentada de estas, dada la aparición de anticuerpos tipo IgG sobre la membrana plaquetaria, dirigidos contra antígenos de dicha membrana. La destrucción de las plaquetas ocurre en los macrófagos esplénicos, como consecuencia de la presencia de receptores para IgG en la membrana de estos macrófagos. Como antes mencionamos la severidad de la enfermedad esta

Tabla I

\section{NIVEL DE EVIDENCIA Y FUERZA DE RECOMENDACIÓN}

\begin{tabular}{ll}
\hline Nivel de evidencia & Descripción \\
\hline Grado I & $\begin{array}{l}\text { Evidencia obtenida a partir de al menos un ensayo clínico aleatorio y control } \\
\text { do. }\end{array}$ \\
Grado II 1 & Evidencia obtenida a partir de ensayos clínicos no aleatorios y bien diseñados \\
Grado II 2 & Evidencia obtenida a partir de estudios de cohorte. \\
Grado II 3 & Evidencia obtenida a partir de múltiples series comparadas en el tiempo, \\
& control. Incluye resultados de experimentos no controlados. \\
Grado III & $\begin{array}{l}\text { Opiniones basadas en experiencias clínicas, estudios descriptivos o informes. } \\
\text { expertos. }\end{array}$ \\
Nivel de evidencia & Fuerza de recomendación \\
III 1 & A: existe adecuada evidencia científica para adoptar una práctica. \\
II 1, II 2 & B: existe cierta evidencia para recomendar la práctica. \\
III & C: hay insuficiente evidencia para recomendar o no recomendar la práctica. \\
II 1, II 2 & D: existe cierta evidencia para no recomendar la práctica. \\
III 1 & E: existe adecuada evidencia científica para no recomendar la práctica.
\end{tabular}


relacionada con los niveles de lgG ligada a las plaquetas. El anticuerpo puede atravesar la placenta, cubrir las membranas fetales y aumentar su destrucción por el sistema retículo endotelial fetal. En esta revisión no se encontraron artículos que mencionen directamente la existencia de riesgo de hemorragia fetal durante el embarazo. Es importante mencionar que en pacientes cuya enfermedad esta en remisión, el feto tiene riesgos de sufrir trombocitopenia por la existencia de anticuerpos a nivel materno, la trombocitopenia neonatal de grado variable se presenta hasta en $50 \%$ de los casos de PTI materno. En madres portadoras de PTI con recién nacido anterior no trombocitopénico, el riesgo para el recién nacido actual es bajo; la trombocitopenia fetal tiene un riesgo de hemorragia intracraneana (HIC) de 1 a $3 \%$, siendo ésta la complicación más temida $(1,2)$.

\section{Clínica y diagnóstico}

Una forma común de identificar un PTI durante el embarazo es debido a la toma de rutina de un hemograma que muestre un recuento plaquetario bajo 150.000 por $\mathrm{mm}^{3}$ en una paciente totalmente asintomática. Los síntomas y signos aparecerán según el nivel plasmático de plaquetas, como se presenta en la Tabla II, y las condiciones asociadas que posea la paciente. Ocasionalmente, las pacientes refieren sangrado mucocutáneo de larga data que se ha exacerbado durante el embarazo. También existe un grupo de pacientes que presentan un PTI de larga data en periodo de remisión o en tratamiento y cursan un embarazo. Lo que no debería ocurrir es el diagnóstico al momento del parto o en una cirugía en que se produciría un sangrado patológico $(1,3)$.

\section{Laboratorio}

En el hemograma encontramos un recuento de plaquetas bajo $150.000 \mathrm{~mm}^{3}$ (el recuento de pla- quetas materno no se correlaciona con el recuento fetal), eritrocitos y leucocitos normales. El mielograma muestra megacariocitos normales o aumentados. Hay presencia de anticuerpos antiplaquetarios circulantes en el $30 \%$ de los casos de PTI. La inmunoglobulina $G$ asociada a las plaquetas (PAlgG), se encuentra elevada hasta en $85 \%$ de los casos de PTI, sin embargo, hasta en $20 \%$ de las trombocitopenias no inmunes se puede encontrar aumentada. En la mayoría de los casos se diagnostica debido a la plaquetopenia, descartando otras causas anteriormente mencionadas (2).

\section{Tratamiento y manejo}

Como hemos visto afecta tanto a la madre como al feto por lo que el tratamiento tiene que ser enfocado hacia ambos.

El manejo de la madre se puede basar en diferentes niveles, partiendo por la terapia esteroidal, cuya droga de elección es la prednisona en dosis habituales de 0,5 a $1 \mathrm{mg} / \mathrm{kg} / \mathrm{día}$ fraccionadas, esto produce una disminución de la fagocitosis por los macrofagos y una disminución de la síntesis de anticuerpos. Se mantiene esta dosis hasta alcanzar un nivel de plaquetas sobre $80.000 / \mathrm{mm}^{3}$ y se disminuye de forma gradual en una semana. Al cabo de dos semanas que no suban los niveles plaquetarios se puede aumentar la dosis a 1,5 a $2 \mathrm{mg} / \mathrm{kg} / \mathrm{día}$ y ver los resultados; se produce respuesta entre un 70 a $90 \%$ de los pacientes con esta terapia de primera línea, pero también existe un porcentaje importante que recidiva cuando se disminuyen las dosis de esteroides $(2,4,5)$.

De segunda línea se puede utilizar inmunoglobulina $G$ previo a una cirugía o parto. La dosis recomendada es de 0,4 g/ $\mathrm{kg} /$ día por 3-5 días, en infusión continua durante 4 a 6 horas, esto produce un bloqueo de los receptores de inmunoglobulina $\mathrm{G}$ en los macrófagos esplénicos produciendo un aumento plaquetario de forma rápida. Solo

Tabla II

MANIFESTACIONES CLÍNICAS DEL PTI SEGÚN RECUENTO PLAQUETARIO

\begin{tabular}{ll}
\hline Recuento de plaquetas & Síntomas y signos \\
\hline 50.000 a $150.000 / \mathrm{mm}^{3}$ & Asintomático \\
10.000 a $50.000 / \mathrm{mm}^{3}$ & Hematomas espontáneos \\
& Menorragia en adolescentes \\
Menor a $10.000 / \mathrm{mm}^{3}$ & Hemorragias en mucosas: epistaxis, gastrointestinales, genitourinarias. \\
& Hemorragia con compromiso vital: sistema nervioso central, etc.
\end{tabular}


en casos extremos durante el embarazo se puede plantear una explenectomía, y de realizarse se prefiere durante el segundo trimestre de la gestación. Excepcionalmente se puede plantear una transfusión de plaquetas en hemorragias graves $(3,5)$.

En el caso del feto la situación es más compleja. Se sugiere conocer de forma directa los niveles de plaquetas a través de una cordocentesis $(2,3)$. Sin esta es imposible predecir el nivel de plaquetas fetales. Por consiguiente se recomienda realizar cordocentesis a las 38 semanas de gestación, si el recuento plaquetario es mayor a 50.000 por $\mathrm{mm}^{3}$ no existiría contraindicación para el parto vaginal y se puede esperar el inicio espontáneo del parto. Si el parto se inicia antes de realizar una cordocentesis se puede realizar un recuento plaquetario de una muestra de sangre capilar de cuero cabelludo fetal cuando la madre tiene 30 más $\mathrm{cm}$ de dilatación cervical $(1,2)$.

\section{Importancia del PTI en el embarazo}

Los riesgos maternos están dados por la posibilidad de hemorragia y tienen relación directa con el grado de plaquetopenia como hemos mencionado anteriormente, puede afectar distintos parénquimas y en cualquier momento del embarazo: a nivel gastrointestinal, de la vía urinaria, intracraneana, intraparto o intraoperatoria y/o postparto, complicaciones anestésicas (hematoma epidural; hemorragia subaracnoidea). La anestesia no esta contraindicada salvo cuando se encuentran niveles de plaquetas menores a $50.000 / \mathrm{mm}^{3}(1,2)$.

\section{¿Que vía de parto utilizar?}

Como hemos dicho anteriormente lo más controvertido y donde menos consenso se ha logrado es sobre la vía de parto cuando tratamos una embarazada con PTI.

En la literatura no encontramos un trabajo prospectivo randomizado (implicancias éticas) con un buen grado de evidencia y fuerza que afirme una vía por sobre otra. Lo que si se encuentra son trabajos de reportes de casos y guías de expertos que recomiendan un manejo por sobre otro, claro está con un nivel de evidencia tipo III-C (3).

Es así como el British Committee for Standards in Haematology en el 2003 presenta una guía para la investigación y manejo del PTI, sugiriendo que las complicaciones neonatales son escasas, siendo la de más importancia HIC como antes se mencionó (3). No se encontró evidencia suficiente como para demostrar la seguridad de la cesárea en disminuir las complicaciones tanto fetales como maternas. Además agregan que la cordonocentesis para medir nivel plaquetario fetal alcanzaría una morbimortalidad de $1-2 \%$. Por lo tanto, existiría un mayor riesgo en realizar una cordocentesis que las complicaciones más graves fetales producto de la trombocitopenia. Así también postulan que la vía de parto debe ser determinada según las condiciones obstétricas.

George et al realizaron una reunión de expertos en 1997 para lograr un consenso sobre el manejo del PTI incluyendo las pacientes embarazadas (4). Sobre esto último recomiendan parto vaginal con niveles plaquetarios maternos entre 10.000-50.000 por $\mathrm{mm}^{3}$ y fetales sobre 20.000 por $\mathrm{mm}^{3}$. Se recomienda optar por cesárea con niveles entre 30.000-50.000 por $\mathrm{mm}^{3}$ y plaquetas fetales menores a 20.000 por $\mathrm{mm}^{3}$. Es así que sugieren que la vía de parto no debe ser elegida debido a los niveles plaquetarios maternos, dado que no logran acuerdo sobre en que punto colocar el nivel de corte. Sobre las plaquetas fetales concluyen que el nivel de corte debería realizarse a 20.000 por $\mathrm{mm}^{3}$, pero sin un ensayo clínico que lo respalde. La toma de estas es controvertido, al igual que el trabajo anterior, refieren que la cordocentesis debe realizarse solo por expertos en centros de referencia, y que las complicaciones (distrés, hemorragia, muerte fetal) son mayores que aquellas producidas por la plaquetopenia. Sobre la muestra de cuero cabelludo, los autores refieren que entre un 25 a $50 \%$ la muestra es insuficiente. La complicación más frecuente es el cefalohematoma y sangrado importante durante el procedimiento. Concluyeron que no existen complicaciones de una vía de parto por sobre la otra, que la vía de parto debe ser de acuerdo a condiciones obstétricas y no al nivel de plaquetas maternas y que la toma de plaquetas fetales no está indicado realizarla puesto que tendría mayores complicaciones, pero si se obtienen debe realizarse cesárea en caso de tener un nivel plasmático menor a $20.000 / \mathrm{mm}^{3}$.

Webert y cols, presentan un trabajo retrospectivo de once años de análisis de pacientes embarazadas con $\mathrm{PTI}$ (5). Obtuvieron un total de 119 casos con plaquetas maternas sobre $50.000 / \mathrm{mm}^{3}$. En $82,4 \%$ de los casos la vía de parto fue vaginal, y $17,6 \%$ por cesárea. No existía diferencia estadística entre los niveles de plaquetas maternos $(p<0,16)$. En 17 pacientes se encontraron niveles plaquetarios menores a $50.000 / \mathrm{mm}^{3}$. Se optó por parto vaginal en $76 \%$ de ellas y $24 \%$ cesárea con niveles de plaquetas entre 3.000 y $39.000 / \mathrm{mm}^{3}$. 
Del total de pacientes se pesquizaron 6 complicaciones maternas, 4 con pérdida de sangre estimada en un litro al momento del parto sin afectar a la paciente, 2 pacientes presentaron sangrado post parto. Se apreció que ninguna de las complicaciones tenía relación con la trombocitopenia. De las complicaciones del recién nacido, uno presentó una hemorragia subependimaria derecha que tampoco la atribuyeron a la trombocitopenia. Concluyeron que la vía de parto debe ser vaginal salvo que la cesárea esté indicada por razones obstétricas.

A nivel nacional, Perucca y cols, realizaron un trabajo retrospectivo entre 1992 y 2002. Se estudiaron 14 casos, dividiéndolos en dos grupos, activos (sobre $100.000 / \mathrm{mm}^{3}$ ) e inactivo (bajo $100.000 / \mathrm{mm}^{3}$ ) (6). En el primer grupo se obtuvieron 8 casos, de los cuales tres fueron por parto vaginal y cinco por cesárea. De los inactivos tres fueron por parto vaginal y tres por cesárea. Se pudo apreciar que las indicaciones de intervención por cesárea fueron por causas obstétricas o por contar con hemocomponentes al momento de interrumpir. Se encontraron complicaciones maternas en dos pacientes, un hematoma vaginal y la otra con un hematoma abdominal que necesitaron drenaje quirúrgico. En cuanto al recién nacido la única complicación que se encontró fue un óbito y un mortineonato en un embarazo gemelar, refieren no tener relación con la trombocitopenia. Concluyen que las complicaciones no tienen relación con la vía de parto, y recomiendan que la indicación de cesárea sea por causas obstétricas.

Sobre la cordocentesis, Song y cols, realizaron junto a expertos mediciones de plaquetas fetales, confirmando que no tienen relación con el nivel plaquetario materno. Tomaron 31 pacientes, 24 de ella tuvieron parto vaginal, siete con cesárea debido a causas obstétricas (7). Del total, 24 pacientes presentaban plaquetas menores a 50.000 por $\mathrm{mm}^{3}$. Se realizó cordocentesis a 16 fetos, ninguno de ellos presentó plaquetas menores a $50.000 / \mathrm{mm}^{3}$, no describieron complicaciones. Sugieren que se debería optar por cesárea si se obtienen niveles plaquetarios fetales menores a $50.000 / \mathrm{mm}^{3}$, pero que la toma de esto no se debería realizar en centros con poca experiencia, y que la incidencia de morbimortalidad del procediento supera las complicaciones fetales, tanto en frecuencia como en gravedad. Por lo tanto la vía de parto debe ser según condiciones obstétricas, dado que las complicaciones del PTI debido al parto vaginal son muy bajas.

Otros estudios concluyen que la vía del parto no tiene relación con las complicaciones, que la HIC alcanzaría cifras entre 1 y $2 \%(8,9)$. La cesárea sin causa obstétrica y la cordocentesis no estarían recomendados $(10,11,12)$. No existirían mayores riesgos para parto vaginal en mujeres con trombocitopenia $(13,14,15)$. La vía de parto no estaría determinada por condiciones asociadas al PTI, sino sólo a condiciones obstétricas $(16,17)$. El Johns Hopkins Manual of Gynecology an Obstetrics, 2nd edition (mayo de 2002), muestran series de $1 \%$ de HIC en recién nacidos de madres con $\mathrm{PTI}$, este último no aumentaría el riesgo de HIC, refieren que no es necesario el recuento de plaquetas para la vía de parto. La HIC sería un evento neonatal y no intraparto.

\section{CONCLUSIONES}

En esta revisión no se encontraron estudios con buen nivel de evidencia que avalara un manejo estandarizado para definir una vía de parto en pacientes portadoras de PTI. El nivel de evidencia y fuerza que se logró encontrar en los distintos trabajos y textos analizados fue III-C (Tabla I). Podemos concluir que la vía del parto se definiría según condiciones obstétricas y no por el nivel de plaquetas fetales, ya que el hecho de realizar alguno de los procedimientos para determinar los niveles plaquetarios fetales estaría relacionado a un mayor riesgo de morbimortalidad que el de la patología misma. Se necesitan trabajos prospectivos randomizados de seguimiento a largo plazo del curso clínico del PTI durante el embarazo para definir una conducta adecuada y que pueda ser recomendada con niveles de evidencia apropiados, sin dejar de lado el tema ético que involucra este hecho.

\section{BIBLIOGRAFÍA}

1. Púrpura trombocitopénico autoinmune. Hallado en http://escuela.med.puc.cl/paginas/Departamentos/ Obstetricia/AltoRiesgo/PTI.html. Acceso el 10 de noviembre de 2005.

2. Madero L, Molina J, Sevilla J. Púrpura Trombocitopénico Idiopático, Controversias. BSCP Can Ped 2001;25(2):291-302.

3. British Committee for Standards in Haematology. Guidelines for the investigation and management of idiopathic thrombocytopenic purpura in adults, children and in pregnancy. $\mathrm{Br} J$ Haematol 2003; 120(4):574-96.

4. George J, Woolf S, Rakob G, Wasser JS, Aledort LM, Ballem PJ, et al. Idiopathic Throbocytopenic Purpura: A practice guideline developed by explicit 
methods for The American Society of Hematology. Blood 1996;88:3-40.

5. Webert K, Mittal R, Sigouin C, Sigouin C, Heddle N, Kelton J. A retrospective 11 year analysis of obstetric patients with ITP. Blood 2003;102(13):4306-11.

6. Perucca E, Muñoz P, Liendo F, Ricci P, Pérez C., Domínguez $\mathrm{C}$, et al. Experiencia y manejo del púrpura trombocitopénico idiopático durante el embarazo. Rev Chil Obstet Ginecol 2003; 68(4):293-99.

7. Song T, Lee J, Kim Y, Choi Y. Low neonatal risk of trombocytopenia in pregnancy associated with ITP. Fetal Diagn Ther 1999;114(4):216-9.

8. Payne SD, Resnik R, Moore TR. Maternal characteristics and risk of severe neonatal thrombocytopenia and intracraneal hemorrhage in pregnancies complicated by autoinmune thrombocytopenia. Am J Obstet Gynecol 1997;177:149-55.

9. Rolbin SH, Abbott D, Musclow E, Papsin F, Lie LM, Freedman J. Epidural anesthesia in pregnant patients with low platelet counts. Obstet Gynecol 1988; 71:918-20.

10. Weiner CP. Cordoscentesis. Obstet Gynecol Clin North Am 1988;15:864-5.

11. Bofill JA, Young RA. Successful pregnancy in a woman with severe factor $X$ deficiency. Obstet Gynecol 1996;88(4 Pt 2):723.
12. Burrows RF, Kelton JG. Fetal thrombocytopenia and its relation to maternal thrombocytopenia. $\mathrm{N} \mathrm{Engl} \mathrm{J}$ Med 1993;329:1463-66.

13. Bussel J, Kaplan C, McFarland J. Working Party on Neonatal Immune Thrombocytopenia of the Neonatal Hemostasis Subcommittee of the Scientific and Standardization Committee of the ISTH. Recommendations for the evaluation and treatment of neonatal autoimmune and alloimmune thrombocytopenia. Thromb Haemost 1991;65: 631-4.

14. Silverman M. Idiopathic Thrombocytopenic purpura, 2005. Hallado en www.emedicine.com /emerg/topic 282.htm. Acceso el 10 de noviembre 2005.

15. Baptista H, Sotelo J, Moreno M, Rosenfeld M. Trombocitopenia gestacional y parto pretérmino. Analisis de la evidencia. Med Sur 2001;8(4):112-6.

16. Payne SD, Resnik R, Moore TR. Maternal characteristics and risk of severe neonatal thrombocytopenia and intracraneal hemorrhage in pregnancies complicated by autoinmune thrombocytopenia. Am J Obstet Gynecol 1997;177:149-55.

17. Olenich M, Schattner E. Postpartum Thrombotic Thrombocytopenic Purpura (TTP) Complicating Pregnancy-associated Inmune Thromboytopenic Purpura (ITP). Ann Intern Med 1994;120:845-7. 